\title{
Diagnostic triad of pulmonary anthracofibrosis in spiral CT scan - a retrospective study
}

\author{
Shahram Kahkouee ${ }^{1 A, B, E}$, Shaghayegh S. Khabbaz ${ }^{2 B, C, E, F}$, Elham Keshavarz ${ }^{3 B, D}$, Arda Kiani ${ }^{4 C, D, F}$, \\ Ghazal Hajinasrollah ${ }^{5 B, E, F}$, Amine Ghaffari Anvar ${ }^{2 B, D, F}$
}

'Department of Radiology, National Research Institute of Tuberculosis and Lung Diseases, Masih Daneshvari Hospital, Shahid Beheshti University of Medical Sciences, Tehran, Iran

${ }^{2}$ Department of Radiology, Shohada-e-Tajrish Hospital, Shahid Beheshti University of Medical Sciences, Tehran, Iran ${ }^{3}$ Department of Radiology, Mahdieh Hospital, Shahid Beheshti University of Medical Sciences, Tehran, Iran

${ }^{4}$ Tracheal Diseases Research Center, Nationah Research institute of Tuberclosis and Lung Disease, Shahid Beheshti University of Medical Sciences, Tehran, Iran ${ }^{5}$ Department of Community Medicine, Shahid Beheshti University of Medical Sciences, Tehran, Iran

\section{Abstract}

Purpose: Anthracosis, a form of pneumoconiosis commonly caused by air pollution and other environmental factors, is a new entity in respiratory disorders. Bronchoscopy and transbronchial lung biopsy (TBLB) are the gold standard of diagnosis. Herein, we evaluated the results of bronchoscopy and chest computed tomography (CT) scans of 187 anthracotic patients.

Material and methods: Between April 2016 and April 2017, 187 cases (99 males, mean age $65 \pm 10.2$ years) who underwent flexible bronchoscopy and TBLB for various indications were considered for this study. CT examinations of these patients were reported as "blind to bronchoscopy results" by two experienced board-certified radiologists.

Results: According to the results of bronchoscopy and TBLB, 100 patients were diagnosed as anthracotic. CT scans confirmed 71 of these cases as anthracosis. Sensitivity, specificity, PPV, NPV, and accuracy of hyperdense non-calcified mediastinal and hilar lymph nodes, known as "brilliant lymph nodes", compared to bronchoscopy were 55\%, $92 \%, 89 \%, 64 \%$, and $72 \%$, respectively (CI: $95 \%$ ). Also, there was a positive correlation between anthracosis and brilliant lymph nodes ( $\mathrm{p}$-value $=0$ ). Analysis of hyper-attenuated lung with bronchoscopy estimated sensitivity, specificity, PPV, NPV, and accuracy gave $41 \%, 94 \%, 89 \%, 58 \%$, and $65 \%$, respectively (CI: $95 \%$ ). The specificity of brilliant lymph nodes, hyper-attenuated lung, and multi-segmental atelectasis as a diagnostic "triad of anthracosis" was $100 \%$.

Conclusions: Based on our analyses, the triad of brilliant lymph nodes, hyper-attenuated lung, and multi-segmental atelectasis was identified as a reliable set of imaging findings for the diagnosis of anthracosis.

Key words: computed tomography, atelectasis, anthracosis, mediastinal lymph nodes.

\section{Introduction}

Anthracosis is an ancient disease with an increasing incidence in industrialised countries characterised by the asymptomatic or mild type of pneumoconiosis occurring following accumulation of carbon in the lungs because of exposure to inhalation of coal dust particles in areas with polluted air [1]. Due to low physical strength and lack of physical vigour, older individuals are more prone to this problem $[2,3]$. Because of the chronic pattern of anthracosis, it is usually misdiagnosed as chronic bronchitis, but

Correspondence address:

Dr. Shaghayegh S. Khabbaz, Department of Radiology, Shohada-e-Tajish Hospital, Tajrish squere, Shahid Beheshti University of Medical Sciences, Tehran, Iran, e-mail:Shaghayegh_khabbaz@yahoo.com

Authors' contribution:

A Study design · B Data collection · C Statistical analysis · D Data interpretation · E Manuscript preparation · F Literature search · G Funds collection 
history of smoking is not considered to be a definite aetiology of the disease [4]. Moreover, anthracosis is not merely localised in bronchi and may spread to the parenchyma and appear as a large mass lesion, with the possibility of invading the regional lymph nodes [5]. Computed tomography (CT) has high sensitivity and specificity for radiological assessment of anthracosis [6]. Previous studies have shown hilar or mediastinal lymphadenopathy in $94 \%$ of subjects, of which $57 \%$ were calcified, pursued by bronchial stenosis and narrowing, with or without atelectasis in $94 \%$ of the cases [7]. The enlarged mediastinal lymph nodes recorded by CT are also observed in the majority of patients with this problem; however, this evidence may also appear in those suffering from metastatic lung cancer, sarcoidosis, chronic heart failure, or pneumoconiosis $[8,9]$. Herein, we evaluate the significance of high attenuated, non-calcified mediastinal and hilar lymph nodes in the diagnosis of anthracosis, and we define the triad of anthracosis with high specificity for diagnosis of anthracosis.

\section{Material and methods}

In total, 455 patients who underwent flexible bronchoscopy and trans-bronchial lung biopsy (TBLB) were prospectively studied. Of those, CT scans of 260 patients were available for reporting, and they were included in the study. Finally, 73 cases were unattainable and indecisive for anthracosis due to severe collapse, pleural effusion, and pulmonary mass, and thus were excluded from the study. Ninety-nine patients were male, and the patients were aged between 60 and 70 years. All patients underwent pulmonary tuberculosis diagnostic workup including standard bronchoalveolar lavage (BAL), sputum smears, and cultures. All examinations were performed with a 16-detector row scanner (Somatom Emotion 16, Siemens Medical Solutions, Erlangen, Germany), collimation: $16 \times 0.75 \mathrm{~mm}$, slice thickness: $5 \mathrm{~mm}$, gantry rotation time: $400 \mathrm{~ms}$, slice interval: $3 \mathrm{ml}$, tube voltage: $120 \mathrm{kV}$, pitch factor: 0.9. All scans were performed from the lung apices to the lung bases, and all images were reviewed using window settings appropriate for lung parenchyma (window width, 1000-1500 HU; window level, -600 to $-700 \mathrm{HU}$ ) and mediastinum (window width, 300-450 HU; window level, 30-50 HU).

Each of the 260 CT examinations was reported as a "blind to bronchoscopy results" interpretation of chest CT by two board-certified radiologists with more than 10 years of experience, with consensus. The following CT findings were considered for the final analysis: multisegmental and uni-segmental atelectasis, bronchiectasis, lung attenuation, bronchial narrowing and peribronchial cuffing, pulmonary nodules, calcified and non-calcified lymph nodes, hyperdense non-calcified lymph nodes, hyperattenuated lung, and consolidation.

The clinicopathological and demographic evidence of the subjects was recorded, and the data analyses were done by the Statistical Package for Social Sciences software version 16.0 (Chicago, IL, USA). Descriptive statistics were presented by mean \pm standard deviation for quantitative variables and number (percentage) for categorical variables. All the categorical comparisons were performed by the $\chi^{2}$ test. $P$-values less than 0.05 were considered statistically significant.

The study protocol was approved by the Masih Daneshvari Hospital, Shahid Beheshti University of Medical Sciences Ethical Committee (Ethical code: IR.SBMU. MSP.REC.1397.113). Informed consent requirement was waived.

\section{Results}

According to the results of bronchoscopy, of the 187 cases studied, with a mean age of $65 \pm 10.2$ years ( $68 \pm 10$ years for anthracosis subjects and $62.4 \pm 10.4$ years for nonanthracosis subjects), 100 patients were diagnosed with anthracosis (58 females). CT scans confirmed the diagnosis in 71 cases, and it was revealed that 17 patients in the control group were mistakenly assigned to anthracosis. Furthermore, in 87 cases (57 males), negative anthracosis was reported as the final diagnosis. In this regard, sensitivity, specificity, positive predictive value (PPV), negative predictive value (NPV), and accuracy of chest CT scans for diagnosis of anthracosis compared to bronchoscopy as the gold standard were $71 \%, 80 \%, 79 \%, 70 \%$, and $75 \%$, respectively. Smokers comprised 35\% of anthracotic patients and $50 \%$ of non-anthracotic patients in the study population.

As observed in $52 \%$ of the patients, bronchial narrowing and peribronchial cuffing were the most prevalent symptoms in chest CT scans. Different symptoms reported by CT scan of the patients with and without anthracosis are summarised in Table 1.

Brilliant lymph nodes and hyper-attenuated lung were two novel signs that were found, in this study, to be the result of anthracosis. Brilliant lymph nodes were outlined as non-calcified, hyperdense mediastinal and hilar lymph nodes with a density higher than normal mediastinal structures such as pulmonary arteries and heart in non-contrast CT scan (Figures 1 and 2). Hyper-attenuated lung is defined as a ground glass appearance and attenuated lung with density higher than muscle tissue (Figures 3 and 4). The sensitivity, specificity, PPV, NPV, and accuracy of brilliant mediastinal and hilar lymph nodes for the diagnosis of anthracosis compared to bronchoscopy were $55 \%, 92 \%, 89 \%, 64 \%$, and $72 \%$, respectively. The positive likelihood ratio (PLR) for these lymph nodes was 6.87 (a valuable sign for ruling in the disease), and the negative likelihood ratio (NLR) was estimated to be 0.48 . Thus, brilliant mediastinal and hilar lymph nodes are suggested as a specific radiologic sign for anthracosis. Also, there was a positive correlation between anthracosis and brilliant lymph nodes $(p<0.001)$. 
Table 1. Comparison of computed tomography (CT) findings in anthracotic and non-anthracotic patients. Significant CT findings are highlighted.

\begin{tabular}{|l|c|c|c|}
\hline CT findings & $\begin{array}{c}\text { Prevalence of findings } \\
\text { in anthracotic patients (\%) }\end{array}$ & $\begin{array}{c}\text { Prevalence of findings } \\
\text { in non-anthracotic patients (\%) }\end{array}$ & $\begin{array}{c}\text { Correlation of findings with } \\
\text { anthracosis (p-value) }\end{array}$ \\
\hline Multisegmental atelectasis & 44 & 11.5 & 0.00 \\
\hline Unisegmental atelectasis & 2 & 5.7 & 0.180 \\
\hline Bronchiectasis & 17 & 36.8 & 0.002 \\
\hline Mosaic lung attenuation & 27 & 13.8 & 0.041 \\
\hline Pulmonary scars and bands & 11 & 11.5 & 0.937 \\
\hline Bronchial narrowing and peribronchial cuffing & 52 & 17.2 & 0.00 \\
\hline Pulmonary nodule & 30 & 20.7 & 0.192 \\
\hline Calcified nodule & 2 & 2.3 & 0.898 \\
\hline Non-calcified nodule & 9 & 14.9 & 0.221 \\
\hline Calcified mediastinal lymph nodes & 27 & 12.6 & 0.012 \\
\hline Non-calcified lymph nodes & 4 & 6.9 & 0.393 \\
\hline Consolidation & 27 & 24.1 & 0.625 \\
\hline Hyper-aeration & 30 & 47.1 & 0.019 \\
\hline Cardiomegaly & 20 & 23 & 0.525 \\
\hline Pulmonary dilatation & 31 & 19.5 & 0.067 \\
\hline Extrapleural fat & 1 & 4.6 & 0.133 \\
\hline Pleural thickening & 1 & 4.6 & 0.133 \\
\hline Cavity & 21 & 6.9 & 0.760 \\
\hline Pleural effusion & & 18.4 & 0.776 \\
\hline
\end{tabular}

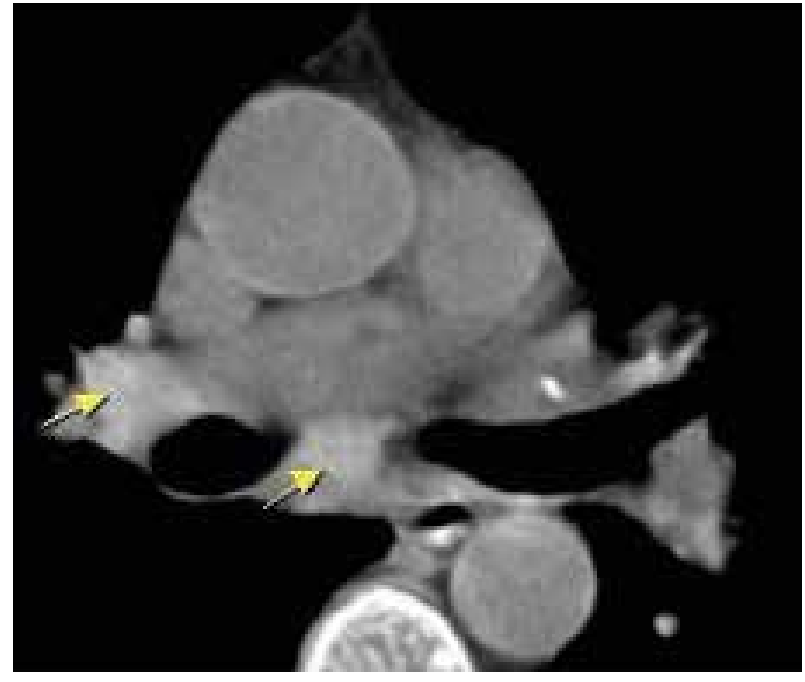

Figure 1. Brilliant lymph nodes. Hyperdense non-calcified mediastinal lymph nodes

The radiologist performed an operator-defined region of interest (ROI) measurement on the hyperdense mediastinal lymph nodes. The ROI was drawn as large as possible, excluding the major heterogeneities such as airways or large blood vessels, and maximum, mean, and standard deviation values were recorded for each patient. The mean maximal lymph node density in the anthracotic patients (54 patients) was $160 \pm 28$, with mean density of $95 \pm 21$ and standard deviation of $27 \pm 10 \mathrm{HU}$. Then, a receiver operating characteristic (ROC) curve was drawn for

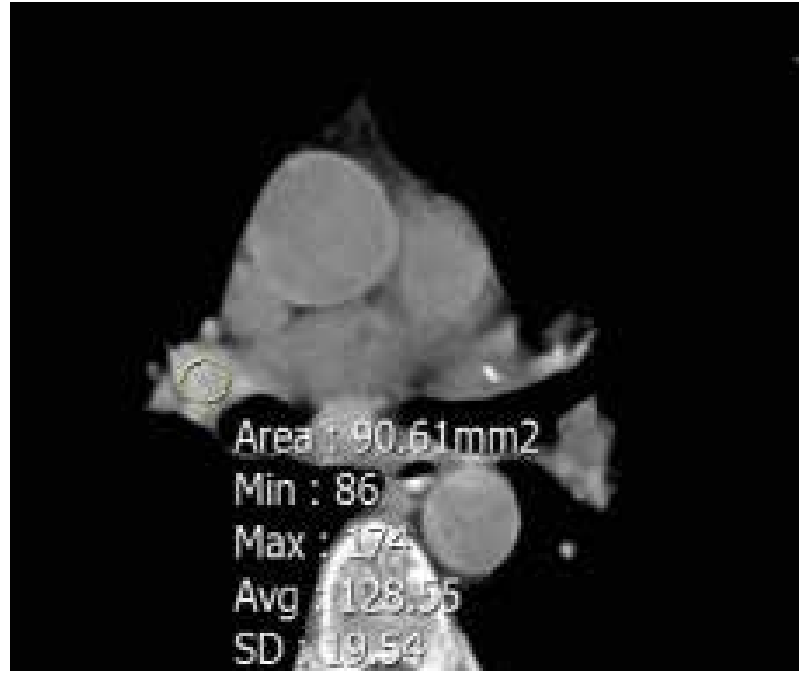

Figure 2. The region of interest (ROI) densitometry of brilliant lymph nodes

maximum density of the lymph node. We report 143 as the best cut-off value for recognising brilliant mediastinal and hilar lymph nodes. Analysis of hyper-attenuated lung, compared to bronchoscopy, yielded sensitivity, specificity, PPV, NPV, and accuracy of $41 \%, 94 \%, 89 \%, 58 \%$, and $65 \%$, respectively.

In this study, the authors assessed brilliant lymph nodes, hyper-attenuated lung, and multisegmental atelectasis as a diagnostic triad for anthracosis, which we termed as the "anthracotic triad" (Figure 5). Based on 


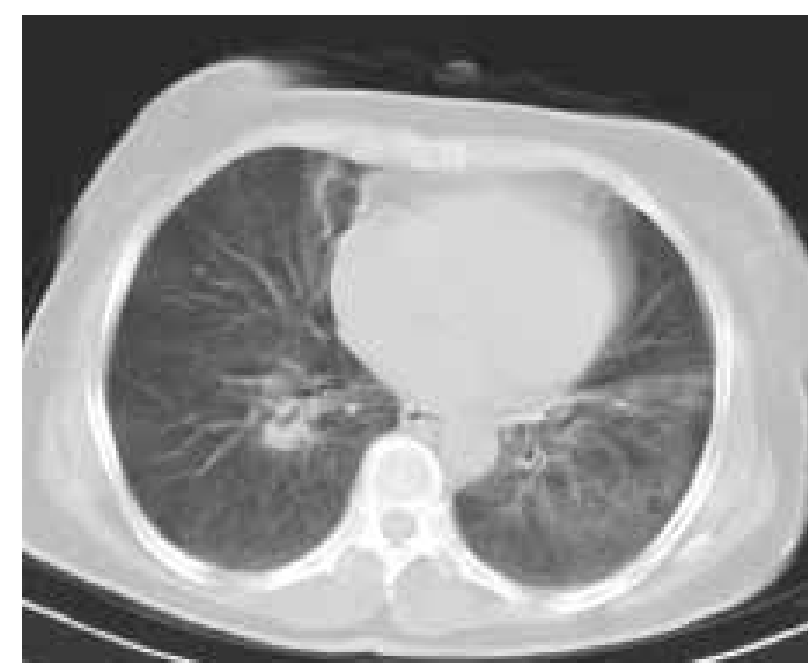

Figure 3. Hyper-attenuated lung

cross-tab, the specificity of this triad was $100 \%$, yielding compatibility with anthracosis when all three symptoms exist simultaneously. Although the sensitivity of this triad is very low, equal to $20 \%$, our findings corroborated a positive correlation between the anthracotic triad and anthracosis.

\section{Discussion}

In accordance with previous studies, our results indicate that in most anthracosis cases, anthracofibrosis patients
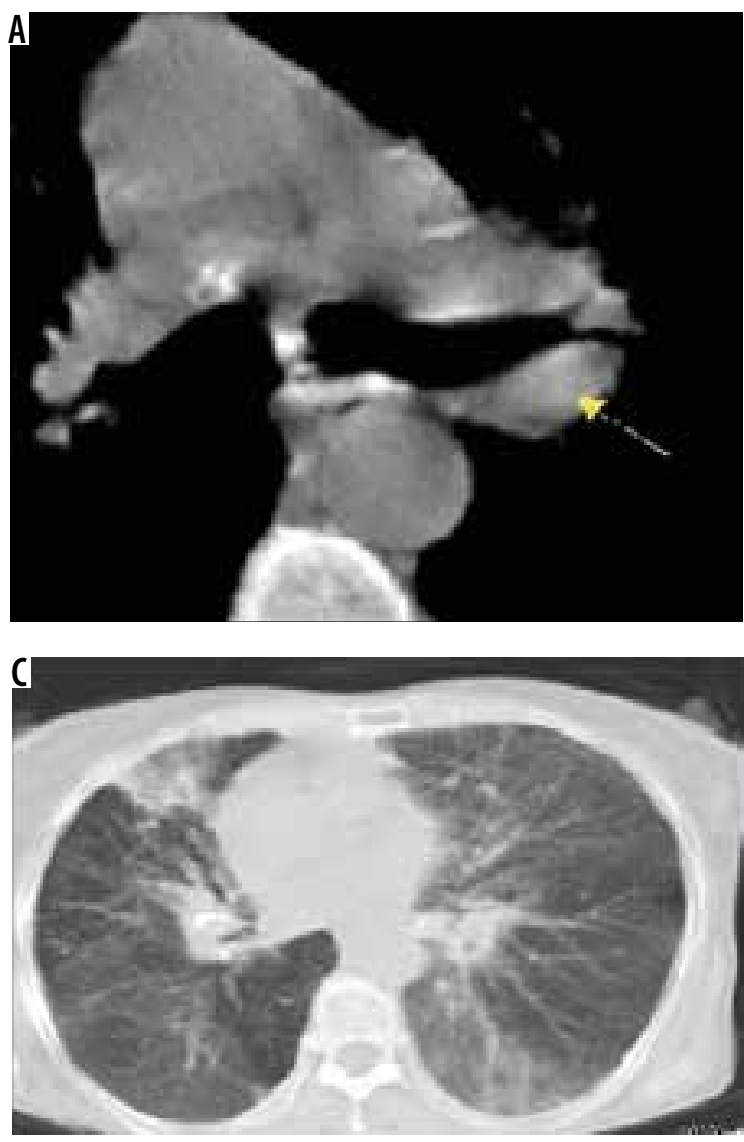

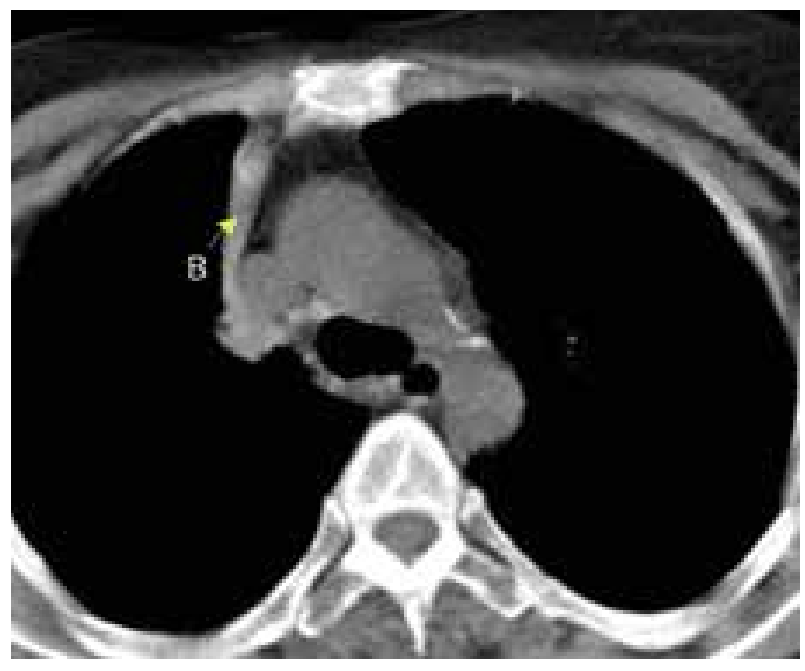

Figure 4. Hyperdense atelectasis

showed radiological abnormalities, while multi-segmental atelectasis, bronchial narrowing, peribronchial cuffing, pulmonary nodule (Figure 6), and consolidation were the most frequent symptoms.

Surprisingly, we observed hyperdense mediastinal lymph nodes and hyperdense parenchyma as two novel symptoms in chest CT scans of anthracotic subjects, with high specificity for diagnosis of this disease, as well as a correlation between the presence of brilliant lymph node, hyper attenuated lung, and atelectasis in CT scan and anthracosis diagnosis.

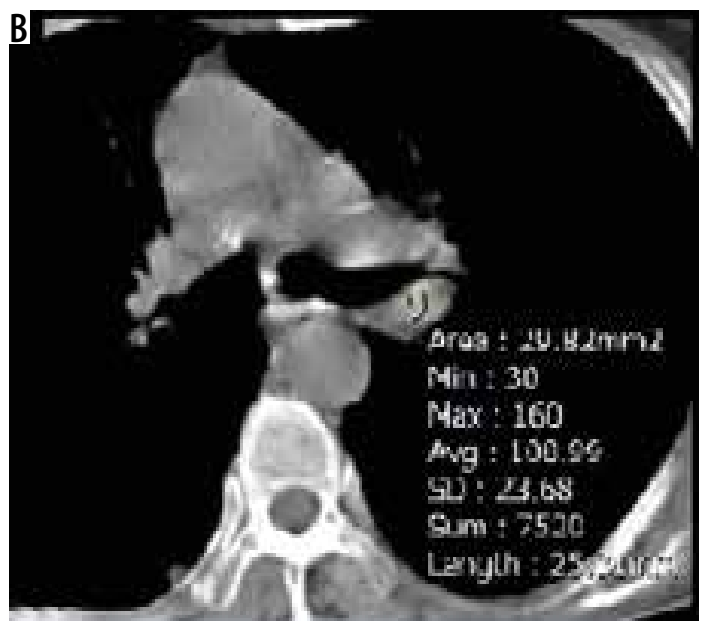

Figure 5. Triad of anthracosis. A) Hyperdense non-calcified mediastinal lymph nodes. B) Region of interest (ROI) densitometry of the mentioned lymph node. C) Hyper-attenuated lung and multi-segmental atelectasis 
The prevalence of anthracosis in women was more than in men, and the mean age of patients in our study was 68 years, which was in accordance with the results of other published studies in Iran [10-12]. This implies that anthracosis is a disease predominantly diagnosed in women and elderly individuals [13].

The initial reports of chest CT imaging showed mediastinal/hilar lymph node enlargement in $94 \%$ of patients, $57 \%$ of which were calcified, followed by bronchial narrowing with or without atelectasis in $94 \%$ of the cases [7]. A study on chest CT findings presented a predominantly higher frequency of lymph node high attenuation (80\%), bronchial wall high attenuation (62\%), bronchial stenosis (48\%), atelectasis (20\%), and mass lesion (14\%) compared to non-anthracotic subjects [2,6]. Singh et al. studied 30 anthracotic cases, and their chest CT scans recorded signs of fibrosis $(43.3 \%)$, consolidation $(33.3 \%)$, cavitation (16.6\%), and mass (46.6\%). Also, $60 \%$ of the cases and $15 \%$ of controls were diagnosed with either old or active pulmonary tuberculosis [14].

The right middle lobe followed by the upper lobes were commonly reported as the most frequently complicated lobes in lung anthracosis $[2,7,11,15]$. In a similar study, central peribronchial soft tissue thickening (63.8\%) causing bronchial narrowing $(63.8 \%)$ or obstruction (19\%) was identified as critical data on chest CT imaging. Multiple bronchial stenosis with simultaneous involvement of two, three, and five bronchi were seen in $21 \%$, $15 \%$, and $3.4 \%$ subjects, respectively [16].

Törün et al. published a description of chest CT scans of anthracotic subjects, in which consolidation, reticulonodular patterns, and masses reported in the CT scans were similar to our results, except for the lower frequency of calcification (22\%) [17]. In another study by Dhar et al., CT scans of 40 anthracotic patients were analysed, of which the most common symptom was multisegmental non-contiguous bronchostenosis observed in $93 \%$ of the cases, with predominant involvement of the right middle lobe. Also, $85 \%$ of the patients showed lymph node enlargement involving hilar, peribronchial, and mediastinal nodes. The nodes were calcified in $91.7 \%$ of the cases, with $58 \%$ showing a pressure effect on adjacent bronchi due to nodal enlargement. Other common symptoms included peribronchial cuffing and bronchial obstruction found in $57 \%$ and $28 \%$, respectively [18]. Hemmati et al. reported hilar or interlobar lymphadenopathy in $80 \%$ (27/34) of their anthracotic patients [19]. Previously, nodal conflu-

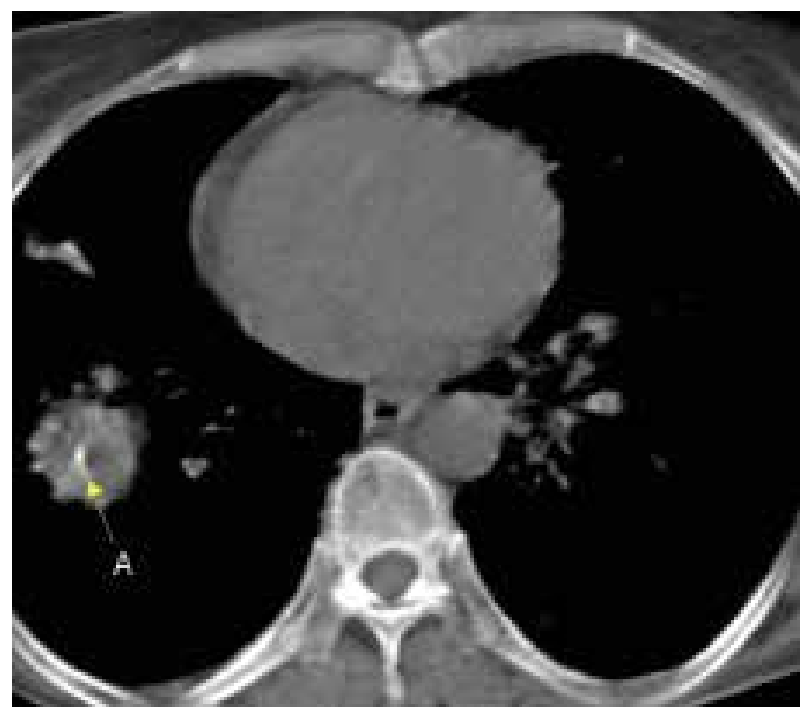

Figure 6. Biopsy-proven anthracotic mass

ence had been assessed as a reliable indicator of anthracosis diagnosis; herein, for the first time, we report an association between brilliant lymph nodes, hyper-attenuated lung, and anthracosis, with high predictive value and accuracy.

However, there were limitations to our study. This was an observational retrospective study, and some clinical evidence is missing, including the history of previous pneumoconiosis or other inflammatory airway diseases. To compensate for this matter, an investigation of a larger cohort of cases would be crucial.

\section{Conclusions}

In conclusion, the triad of brilliant lymph nodes, hyperattenuated lung, and multi segmental atelectasis, identified by chest CT scan imaging, is introduced as a reliable sign for the diagnosis of anthracosis.

\section{Acknowledgements}

The authors would like to thank all the staff of the Pathology and Radiology section of Masih Daneshvari Hospital for their support and assistance with this project.

\section{Disclosure}

The authors declare no conflict of interests.

Reference

1. Mirsadraee M, Saeedi P. Anthracosis of lung: evaluation of potential underlying causes. J Bronchology Interv Pulmonol 2005; 12: 84-87.

2. Mirsadraee M, Asna-Ashari A, Attaran D, et al. Bronchial anthracosis: a new diagnosis for benign mass lesions of the lung. Tanaffos 2013; 12: 10-18.
3. Mirsadraee M, Saeedi P. Anthracosis of the lung: evaluation of potential causes. Iran J Med Sci 2015; 30: 190-193.

4. Kim YJ, Jung CY, Shin HW, Lee BK. Biomass smoke induced bronchial anthracofibrosis: presenting features and clinical course. Respiratory Med 2009; 103: 757-765. 
5. Cserni G. Misidentification of an axillary sentinel lymph node due to anthracosis. Eur J Surg Oncol 1998; 24: 168.

6. Mirsadraee M. Anthracosis of the lungs: etiology, clinical manifestations and diagnosis: a review. Tanaffos 2014; 13: 1-13.

7. Kim HY, Im J-G, Goo JM, et al. Bronchial anthracofibrosis (inflammatory bronchial stenosis with anthracotic pigmentation) CT findings. Am J Roentgenol 2000; 174: 523-527.

8. Kirchner J, Broll M, Müller P, et al. Mediastinal lymph node staging by EBUS-TBNA and CT: characteristics of lymph node enlargement caused by anthracosis and other benign conditions. Int J Diag Imaging 2014; 1: 64-73.

9. Alavi A, Akhoundzadeh N, Karkan MF. Sarcoidosis, a report from Guilan (an Iranian Northen province) (2001-09). Sarcoidosis Vasc Diffuse Lung Dis 2015; 31: 282-288.

10. Ghanei M, Aslani J, Peyman M, et al. Bronchial anthracosis: a potent clue for diagnosis of pulmonary tuberculosis. Oman Med J 2011; 26: 19-22.

11. Fekri MS, Lashkarizadeh MR, Kardoost AH, Shokoohi M. Bronchial anthracosis and pulmonary tuberculosis. Tanaffos 2010; 9: 21-25.

12. Amoli K. Anthracotic airways disease: Report of 102 cases. 2009. Tanaffos 2009; 8: 14-22.
13. Pazoki M, Goodarzi HM, Taheri AH, et al. Prevalence of tuberculosis in patients with anthracosis: study on 150 subjects. Arch Iran Med 2012; 15: 128-130.

14. Singh V, Meena H, Bairwa R, et al. Clinico-radiological profile and risk factors in patients with anthracosis. Lung India 2015; 32: 102106.

15. Lee HS, Maeng JH, Park PG, et al. Clinical features of simple bronchial anthracofibrosis which is not associated with tuberculosis. Tuberc Respir Dis 2002; 53: 510-518.

16. Kahkouee S, Pourghorban R, Bitarafan M, et al. Imaging findings of isolated bronchial anthracofibrosis: a computed tomography analysis of patients with bronchoscopic and histologic confirmation. Arch Bronconeumol 2015; 51: 322-327.

17. Törün T, Gungor G, Ozmen I, et al. Bronchial anthracostenosis in patients exposed to biomass smoke. Turkish Respir J 2007; 8: 48-51.

18. Dhar A, Sikund K, Lall A, Aggarwal B. Radiological spectrum of anthracofibrosis: a series of 40 patients with computed tomography, bronchoscopy, and biopsy. Indian J Radiol Imaging 2017; 27: 397-403.

19. Hemmati SH, Shahriar M, Molaei NA. What causes anthracofibrosis? Either tuberculosis or smoke. Pak J Med Sci 2008; 24: 395-398. 